OPEN $\curvearrowright$ ACCESS

ISSN 2056-9890

\section{Crystal structure of 3-bromo-4-dimethyl- amino-1-methyl-1,2,4-triazol-5(4H)-one}

\section{Gerhard Laus,* Thomas Gelbrich, Klaus Wurst and Herwig Schottenberger}

Faculty of Chemistry and Pharmacy, University of Innsbruck, 6020 Innsbruck, Austria. ${ }^{*}$ Correspondence e-mail: gerhard.laus@uibk.ac.at

Received 27 November 2014; accepted 1 December 2014

Edited by L. Farrugia, University of Glasgow, Scotland

The title compound, $\mathrm{C}_{5} \mathrm{H}_{9} \mathrm{BrN}_{4} \mathrm{O}$, was obtained as a minor byproduct in the synthesis of 4-dimethylamino-1-methyl-1,2,4triazolin-5-one. Except for the methyl groups of the 4dimethylamino moiety, all the non- $\mathrm{H}$ atoms lie on a crystallographic mirror plane." In the crystal, the molecules are linked by $\mathrm{C}-\mathrm{Br} \cdots \mathrm{O}=\mathrm{C}$ interactions $[\mathrm{Br} \cdots \mathrm{O}=2.877$ (2) $\AA$, $\left.\mathrm{C}-\mathrm{Br} \cdots \mathrm{O}=174.6(1)^{\circ}\right]$ into infinite chains in the $c$-axis direction.

Keywords: crystal structure; 1,2,4-triazol-5(4H)-one; $\mathrm{Br} \cdots \mathrm{O}=\mathrm{C}$ interactions; halogen interactions.

CCDC reference: 1036852

\section{Related literature}

For synthesis of related 4-amino-1-methyl-1,2,4-triazolin-5ones, see: Kröger et al. (1965). For related structures with $\mathrm{Br} \cdots \mathrm{O}=\mathrm{C}$ interactions, see: 5-bromopyrimidin-2-one (Yathirajan et al., 2007); 3,5-dibromopyran-2-one (Reus et al., 2012); N-bromosaccharin (Dolenc \& Modec, 2009); Nbromosuccinimide (Jabay et al., 1977); dibromantin (Kruszynski, 2007). For the theory of halogen interactions, see: Awwadi et al. (2006). For details of the synthesis, see: Schwärzler et al. (2009).<smiles>CN(C)n1c(Br)nn(C)c1=O</smiles>

\section{Experimental}

\subsection{Crystal data}

$\mathrm{C}_{5} \mathrm{H}_{9} \mathrm{BrN}_{4} \mathrm{O}$

$M_{r}=221.07$

Monoclinic, $C 2 / m$

$a=15.1993(6) \AA$

$b=6.9377(4) \AA$

$c=7.8771(7) \AA$

$\beta=93.869(3)^{\circ}$

$$
\begin{aligned}
& V=828.73(9) \AA^{3} \\
& Z=4 \\
& \text { Mo } K \alpha \text { radiation } \\
& \mu=4.91 \mathrm{~mm}^{-1} \\
& T=233 \mathrm{~K} \\
& 0.09 \times 0.08 \times 0.07 \mathrm{~mm}
\end{aligned}
$$

\subsection{Data collection}

Nonius KappaCCD diffractometer 2310 measured reflections 806 independent reflections

734 reflections with $I>2 \sigma(I)$ $R_{\text {int }}=0.034$

\subsection{Refinement}

$R\left[F^{2}>2 \sigma\left(F^{2}\right)\right]=0.028$

$w R\left(F^{2}\right)=0.065$

$S=1.07$

806 reflections

75 parameters

6 restraints
Data collection: DENZO (Otwinowski \& Minor, 1997) and COLLECT (Hooft, 1998); cell refinement: DENZO and COLLECT; data reduction: $D E N Z O$ and $C O L L E C T$; program(s) used to solve structure: SHELXS97 (Sheldrick, 2008); program(s) used to refine structure: SHELXL2014 (Sheldrick, 2008); molecular graphics: Mercury (Macrae et al., 2006) and ORTEP-3 for Windows (Farrugia, 2012); software used to prepare material for publication: publCIF (Westrip, 2010).

Supporting information for this paper is available from the IUCr electronic archives (Reference: FJ2686).

\title{
References
}

Awwadi, F. F., Willett, R. D., Peterson, K. A. \& Twamley, B. (2006). Chem. Eur. J. 12, 8952-8960.

Dolenc, D. \& Modec, B. (2009). New J. Chem. 33, 2344-2349.

Farrugia, L. J. (2012). J. Appl. Cryst. 45, 849-854.

Hooft, R. W. W. (1998). COLLECT. Nonius BV, Delft, The Netherlands.

Jabay, O., Pritzkow, H. \& Jander, J. (1977). Z. Naturforsch. Teil B, 32, 14161420.

Kröger, C.-F., Hummel, L., Mutscher, M. \& Beyer, H. (1965). Chem. Ber. 98, 3025-3033.

Kruszynski, R. (2007). Acta Cryst. C63, o389-o391.

Macrae, C. F., Edgington, P. R., McCabe, P., Pidcock, E., Shields, G. P., Taylor, R., Towler, M. \& van de Streek, J. (2006). J. Appl. Cryst. 39, 453-457.

Otwinowski, Z. \& Minor, W. (1997). Methods in Enzymology, Vol. 276, Macromolecular Crystallography, Part A, edited by C. W. Carter Jr \& R. M. Sweet, pp. 307-326. New York: Academic Press.

Reus, C., Liu, N.-W., Bolte, M., Lerner, H.-W. \& Wagner, M. (2012). J. Org. Chem. 77, 3518-3523.

Schwärzler, A., Laus, G., Kahlenberg, V., Wurst, K., Gelbrich, T., Kreutz, C., Kopacka, H., Bonn, G. \& Schottenberger, H. (2009). Z. Naturforsch. Teil B, 64, 603-616.

Sheldrick, G. M. (2008). Acta Cryst. A64, 112-122.

Westrip, S. P. (2010). J. Appl. Cryst. 43, 920-925.

Yathirajan, H. S., Narayana, B., Ashalatha, B. V., Sarojini, B. K. \& Bolte, M. (2007). Acta Cryst. E63, o923-o924. 


\title{
supporting information
}

Acta Cryst. (2015). E71, o23 [https://doi.org/10.1107/S205698901402636X]

Crystal structure of 3-bromo-4-dimethylamino-1-methyl-1,2,4-triazol-5(4H)-one

\author{
Gerhard Laus, Thomas Gelbrich, Klaus Wurst and Herwig Schottenberger
}

\section{S1. Comment}

Triazolinones are of relevance due to their wide range of pesticidal activities. The molecular structure of 3-bromo-4-(dimethylamino)-1-methyl-1,2,4-triazolin-5-one is shown in Figure 1. The triazole rings are located in the crystallographic mirror plane (Figure 2), whereas the $\mathrm{C} 4$ methyl groups are situated out of this plane. The molecules are linked by short intermolecular $\mathrm{C}-\mathrm{Br} \cdots \mathrm{O}=\mathrm{C}$ contacts into infinite chains in the direction of the $c$ axis (Figure 3). The $\mathrm{Br} \cdots \mathrm{O}$ distance of 2.877 (2) $\AA$ is significantly shorter than the sum of van der Waals radii. Theoretical calculations predicted negative ring and positive end cap domains of halogen atoms due to their polarizability (Awwadi et al., 2006). The almost linear C$\mathrm{Br} \cdots \mathrm{O}$ angle of $174.6(1)^{\circ}$ indicates an interaction involving the positive end cap of the $\mathrm{Br}$ atom. Thus, the $\mathrm{Br}$ atom acts as an electron-acceptor (X-bond donor) in this case.

\section{S2. Experimental}

The title compound was obtained as a minor by-product in the synthesis of 4-(dimethylamino)-1-methyl-1,2,4-triazolin-5one by hydrolysis of 5-bromo-4-(dimethylamino)-1-methyl-1,2,4-triazolium hexafluorophosphate (Schwärzler et al., 2009) in $\mathrm{MeOH} / \mathrm{H}_{2} \mathrm{O}$. It is assumed that the 5-bromo compound was contaminated with a trace of the corresponding 3,5dibromo compound which resulted in the formation of the present 3-bromo-1,2,4-triazolin-5-one.

\section{S3. Refinement}

The $\mathrm{H}$ atoms were identified in a difference map and those of the $\mathrm{C} 4$ methyl group were idealized and included as rigid groups, allowed to rotate but not tip $(\mathrm{C}-\mathrm{H}=0.97 \AA)$. The $\mathrm{C} 3$ methyl group was found to be disordered over two orientations related by mirror symmetry. Its $\mathrm{H}$ positions were refined with restrained $\mathrm{C}-\mathrm{H}$ and $\mathrm{H} \cdots \mathrm{H}$ distances of 0.97 (1) $\AA$ and 1.58 (2) $\AA$, respectively. The $U_{\text {iso }}$ parameters of all $\mathrm{H}$ atoms were set to $1.5 U_{\text {eq }}(\mathrm{C})$ of the parent carbon atom. 


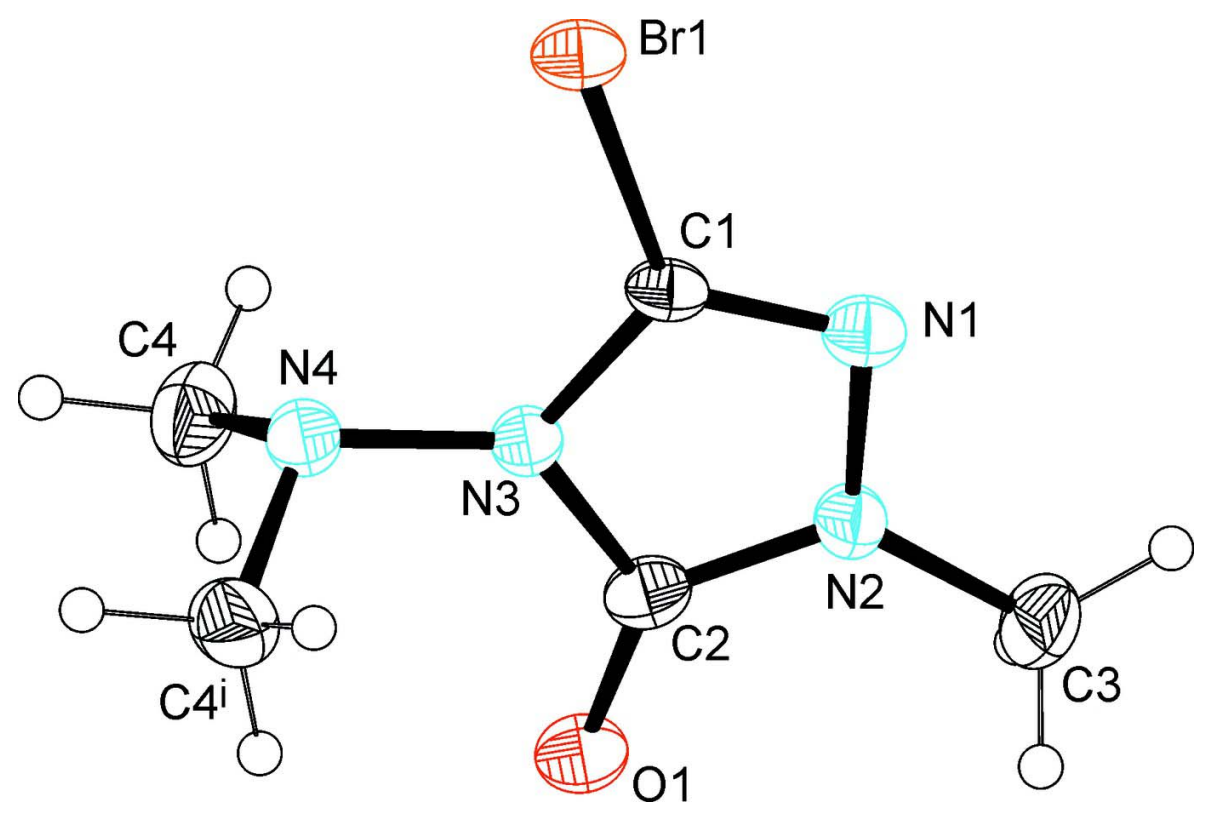

Figure 1

The molecular structure of the title compound, with atom labels and 50\% probability displacement ellipsoids for non- $\mathrm{H}$ atoms. One component of the disordered C3 methyl group has been omitted for clarity. Symmetry code (i): $x,-y, z$. 


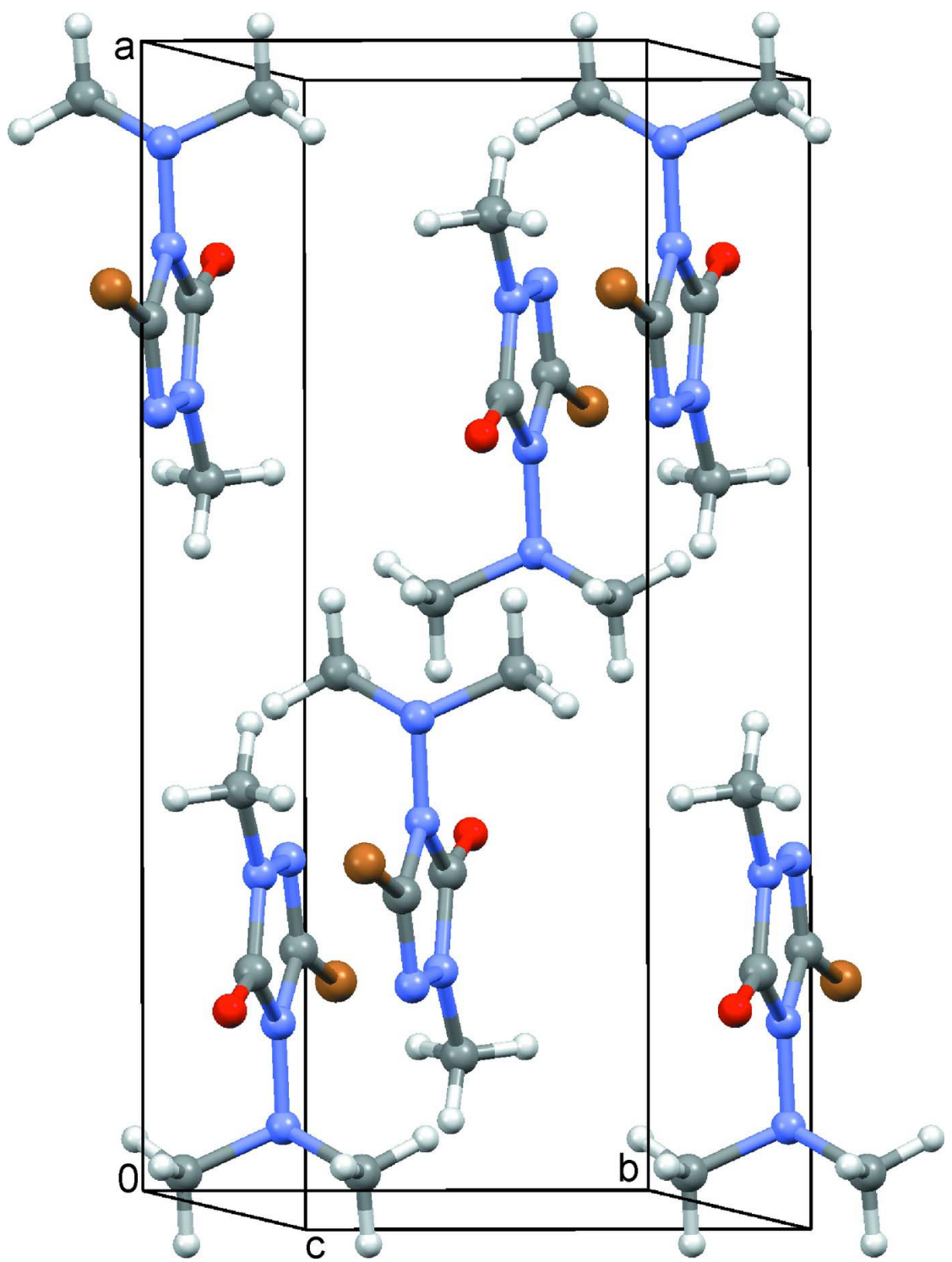

Figure 2

Arrangement of the triazole rings parallel to the $a c$ plane. One component of the disordered C3 methyl group has been omitted for clarity. 


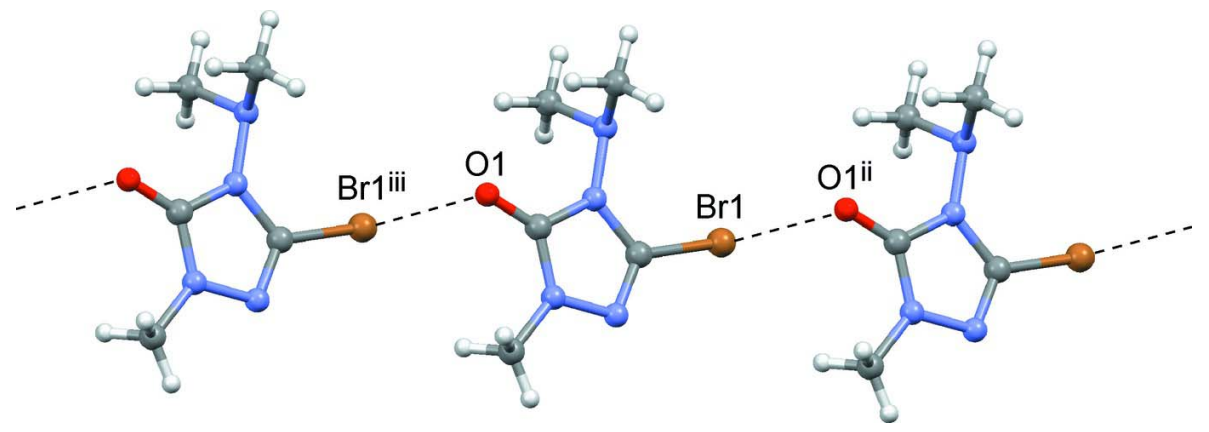

Figure 3

Infinite chains of molecules linked by $\mathrm{Br} \cdots \mathrm{O}$ interactions. One component of the disordered $\mathrm{C} 3$ methyl group has been omitted for clarity. Symmetry code (ii): $x, y, 1+z$; (iii): $x, y,-1+z$.

3-Bromo-4-dimethylamino-1-methyl-1,2,4-triazol-5(4H)-one

\section{Crystal data}

$\mathrm{C}_{5} \mathrm{H}_{9} \mathrm{BrN}_{4} \mathrm{O}$

$M_{r}=221.07$

Monoclinic, $C 2 / m$

$a=15.1993(6) \AA$

$b=6.9377(4) \AA$

$c=7.8771(7) \AA$

$\beta=93.869(3)^{\circ}$

$V=828.73(9) \AA^{3}$

$Z=4$

\section{Data collection}

Nonius KappaCCD

diffractometer

Radiation source: fine-focus sealed tube

Graphite monochromator

phi and $\omega$ scans

2310 measured reflections

806 independent reflections

\section{Refinement}

Refinement on $F^{2}$

Least-squares matrix: full

$R\left[F^{2}>2 \sigma\left(F^{2}\right)\right]=0.028$

$w R\left(F^{2}\right)=0.065$

$S=1.07$

806 reflections

75 parameters

6 restraints
$F(000)=440$

$D_{\mathrm{x}}=1.772 \mathrm{Mg} \mathrm{m}^{-3}$

Mo $K \alpha$ radiation, $\lambda=0.71073 \AA$

Cell parameters from 3066 reflections

$\theta=1.0-25.0^{\circ}$

$\mu=4.91 \mathrm{~mm}^{-1}$

$T=233 \mathrm{~K}$

Prism, colorless

$0.09 \times 0.08 \times 0.07 \mathrm{~mm}$

734 reflections with $I>2 \sigma(I)$

$R_{\text {int }}=0.034$

$\theta_{\max }=25.1^{\circ}, \theta_{\min }=2.6^{\circ}$

$h=-13 \rightarrow 18$

$k=-8 \rightarrow 8$

$l=-9 \rightarrow 8$

Hydrogen site location: difference Fourier map

$\mathrm{H}$ atoms treated by a mixture of independent

and constrained refinement

$w=1 /\left[\sigma^{2}\left(F_{\mathrm{o}}^{2}\right)+(0.033 P)^{2}+0.5344 P\right]$

where $P=\left(F_{\mathrm{o}}^{2}+2 F_{\mathrm{c}}^{2}\right) / 3$

$(\Delta / \sigma)_{\max }<0.001$

$\Delta \rho_{\max }=0.50$ e $\AA^{-3}$

$\Delta \rho_{\min }=-0.44$ e $\AA^{-3}$

Special details

Geometry. All e.s.d.'s (except the e.s.d. in the dihedral angle between two 1.s. planes) are estimated using the full covariance matrix. The cell e.s.d.'s are taken into account individually in the estimation of e.s.d.'s in distances, angles and torsion angles; correlations between e.s.d.'s in cell parameters are only used when they are defined by crystal symmetry. An approximate (isotropic) treatment of cell e.s.d.'s is used for estimating e.s.d.'s involving 1.s. planes. 
Fractional atomic coordinates and isotropic or equivalent isotropic displacement parameters $\left(\hat{A}^{2}\right)$

\begin{tabular}{llllll}
\hline & $x$ & $y$ & $z$ & $U_{\text {iso }} * / U_{\mathrm{eq}}$ & Occ. $(<1)$ \\
\hline Br1 & $0.22218(3)$ & 0.0000 & $0.18788(4)$ & $0.03459(19)$ & \\
O1 & $0.17449(19)$ & 0.0000 & $-0.4649(3)$ & $0.0426(8)$ & \\
N1 & $0.3186(2)$ & 0.0000 & $-0.1029(4)$ & $0.0319(8)$ & \\
N2 & $0.2997(2)$ & 0.0000 & $-0.2784(4)$ & $0.0302(8)$ & \\
N3 & $0.1742(2)$ & 0.0000 & $-0.1669(4)$ & $0.0282(7)$ & \\
N4 & $0.0841(2)$ & 0.0000 & $-0.1382(4)$ & $0.0329(8)$ & \\
C1 & $0.2418(3)$ & 0.0000 & $-0.0415(4)$ & $0.0269(9)$ & \\
C2 & $0.2125(3)$ & 0.0000 & $-0.3221(4)$ & $0.0322(10)$ & \\
C3 & $0.3694(3)$ & 0.0000 & $-0.3938(6)$ & $0.0445(11)$ & 0.5 \\
H3A & $0.352(3)$ & $-0.069(5)$ & $-0.497(4)$ & $0.067 *$ & 0.5 \\
H3B & $0.4248(19)$ & $-0.047(6)$ & $-0.343(6)$ & $0.067 *$ & \\
H3C & $0.375(3)$ & $0.136(2)$ & $-0.420(6)$ & $0.067 *$ & \\
C4 & $0.04175(19)$ & $0.1766(5)$ & $-0.2044(4)$ & $0.0478(8)$ & \\
H4A & 0.0726 & 0.2877 & -0.1550 & $0.072 *$ & \\
H4B & -0.0192 & 0.1791 & -0.1750 & $0.072 *$ & \\
H4C & 0.0438 & 0.1801 & -0.3272 & $0.072 *$ & \\
& & & & & \\
\hline
\end{tabular}

Atomic displacement parameters $\left(\AA^{2}\right)$

\begin{tabular}{lllllll}
\hline & $U^{11}$ & $U^{22}$ & $U^{33}$ & $U^{12}$ & $U^{13}$ & $U^{23}$ \\
\hline Br1 & $0.0454(3)$ & $0.0368(3)$ & $0.0211(3)$ & 0.000 & $-0.00047(17)$ & 0.000 \\
O1 & $0.0429(17)$ & $0.067(2)$ & $0.0181(14)$ & 0.000 & $0.0024(12)$ & 0.000 \\
N1 & $0.037(2)$ & $0.0330(19)$ & $0.0255(17)$ & 0.000 & $-0.0004(14)$ & 0.000 \\
N2 & $0.0279(19)$ & $0.0367(19)$ & $0.0261(17)$ & 0.000 & $0.0020(13)$ & 0.000 \\
N3 & $0.0257(17)$ & $0.0392(19)$ & $0.0197(16)$ & 0.000 & $0.0019(12)$ & 0.000 \\
N4 & $0.0290(18)$ & $0.044(2)$ & $0.0252(17)$ & 0.000 & $0.0013(13)$ & 0.000 \\
C1 & $0.035(2)$ & $0.027(2)$ & $0.019(2)$ & 0.000 & $-0.0026(16)$ & 0.000 \\
C2 & $0.040(3)$ & $0.032(2)$ & $0.025(2)$ & 0.000 & $0.0051(18)$ & 0.000 \\
C3 & $0.036(3)$ & $0.063(3)$ & $0.036(2)$ & 0.000 & $0.0124(19)$ & 0.000 \\
C4 & $0.0402(19)$ & $0.059(2)$ & $0.0445(18)$ & $0.0125(15)$ & $0.0051(14)$ & $0.0066(17)$ \\
\hline
\end{tabular}

Geometric parameters $\left(\AA,{ }^{\circ}\right)$

\begin{tabular}{llll}
\hline $\mathrm{Br} 1-\mathrm{C} 1$ & $1.851(4)$ & $\mathrm{N} 4-\mathrm{C} 4$ & $1.464(4)$ \\
$\mathrm{O} 1-\mathrm{C} 2$ & $1.230(4)$ & $\mathrm{N} 4-\mathrm{C} 4 \mathrm{i}$ & $1.464(4)$ \\
$\mathrm{N} 1-\mathrm{C} 1$ & $1.292(5)$ & $\mathrm{C} 3-\mathrm{H} 3 \mathrm{~A}$ & $0.967(10)$ \\
$\mathrm{N} 1-\mathrm{N} 2$ & $1.392(5)$ & $\mathrm{C} 3-\mathrm{H} 3 \mathrm{~B}$ & $0.965(10)$ \\
$\mathrm{N} 2-\mathrm{C} 2$ & $1.346(5)$ & $\mathrm{C} 3-\mathrm{H} 3 \mathrm{C}$ & $0.969(10)$ \\
$\mathrm{N} 2-\mathrm{C} 3$ & $1.442(5)$ & $\mathrm{C} 4-\mathrm{H} 4 \mathrm{~A}$ & 0.9700 \\
$\mathrm{~N} 3-\mathrm{C} 1$ & $1.377(4)$ & $\mathrm{C} 4-\mathrm{H} 4 \mathrm{~B}$ & 0.9700 \\
$\mathrm{~N} 3-\mathrm{C} 2$ & $1.389(5)$ & $\mathrm{C} 4-\mathrm{H} 4 \mathrm{C}$ & 0.9700 \\
$\mathrm{~N} 3-\mathrm{N} 4$ & $1.403(4)$ & & \\
& & & \\
$\mathrm{Br} 1 \cdots \mathrm{O} 1^{\mathrm{ii}}$ & $2.876(3)$ & &
\end{tabular}




$\begin{array}{llll}\mathrm{C} 1-\mathrm{N} 1-\mathrm{N} 2 & 103.9(3) & \mathrm{O} 1-\mathrm{C} 2-\mathrm{N} 3 & 127.3(4) \\ \mathrm{C} 2-\mathrm{N} 2-\mathrm{N} 1 & 112.8(3) & \mathrm{N} 2-\mathrm{C} 2-\mathrm{N} 3 & 103.8(3) \\ \mathrm{C} 2-\mathrm{N} 2-\mathrm{C} 3 & 126.2(3) & \mathrm{N} 2-\mathrm{C} 3-\mathrm{H} 3 \mathrm{~A} & 111(4) \\ \mathrm{N} 1-\mathrm{N} 2-\mathrm{C} 3 & 121.0(3) & \mathrm{N} 2-\mathrm{C} 3-\mathrm{H} 3 \mathrm{~B} & 113(3) \\ \mathrm{C} 1-\mathrm{N} 3-\mathrm{C} 2 & 107.1(3) & \mathrm{H} 3 \mathrm{~A}-\mathrm{C} 3-\mathrm{H} 3 \mathrm{~B} & 111(2) \\ \mathrm{C} 1-\mathrm{N} 3-\mathrm{N} 4 & 125.0(3) & \mathrm{N} 2-\mathrm{C} 3-\mathrm{H} 3 \mathrm{C} & 102(4) \\ \mathrm{C} 2-\mathrm{N} 3-\mathrm{N} 4 & 127.9(3) & \mathrm{H} 3 \mathrm{~A}-\mathrm{C} 3-\mathrm{H} 3 \mathrm{C} & 109(2) \\ \mathrm{N} 3-\mathrm{N} 4-\mathrm{C} 4 & 110.6(2) & \mathrm{H} 3 \mathrm{~B}-\mathrm{C} 3-\mathrm{H} 3 \mathrm{C} & 110(2) \\ \mathrm{N} 3-\mathrm{N} 4-\mathrm{C} 4 & 110.6(2) & \mathrm{N} 4-\mathrm{C} 4-\mathrm{H} 4 \mathrm{~A} & 109.5 \\ \mathrm{C} 4-\mathrm{N} 4-\mathrm{C} 4 & 113.7(3) & \mathrm{N} 4-\mathrm{C} 4-\mathrm{H} 4 \mathrm{~B} & 109.5 \\ \mathrm{~N} 1-\mathrm{C} 1-\mathrm{N} 3 & 112.4(3) & \mathrm{H} 4 \mathrm{~A}-\mathrm{C} 4-\mathrm{H} 4 \mathrm{~B} & 109.5 \\ \mathrm{~N} 1-\mathrm{C} 1-\mathrm{Br} 1 & 125.0(3) & \mathrm{N} 4-\mathrm{C} 4-\mathrm{H} 4 \mathrm{C} & 109.5 \\ \mathrm{~N} 3-\mathrm{C} 1-\mathrm{Br} 1 & 122.6(3) & \mathrm{H} 4 \mathrm{~A}-\mathrm{C} 4-\mathrm{H} 4 \mathrm{C} & 109.5 \\ \mathrm{O} 1-\mathrm{C} 2-\mathrm{N} 2 & 128.9(4) & \mathrm{H} 4 \mathrm{~B}-\mathrm{C} 4-\mathrm{H} 4 \mathrm{C} & 109.5\end{array}$

Symmetry codes: (i) $x,-y, z$; (ii) $x, y, z+1$. 